\title{
Control de presión arterial y lípidos y riesgo coronario en la población hipertensa asistida en Atención Primaria en España. Estudio PRESCOT
}

\author{
Blood pressure and lipid control and coronary risk in the hypertensive population \\ attended in Primary Care setting in Spain. The PRESCOT study
}

\author{
V. Barrios, C. Escobar, J.L. Llisterri, A. Calderón, E. Alegría, J. Muñiz, A. Matalí
}

\begin{abstract}
Objetivo y métodos. El objetivo de este trabajo, de diseño transversal y multicéntrico, fue conocer el perfil de riesgo coronario de los pacientes hipertensos que acuden a la consulta de Atención Primaria, y evaluar si el control de presión arterial y de colesterol LDL podía variar según el grupo de riesgo coronario ATP-III. Se consideró buen control de presión arterial cifras menores a 140/90 mmHg $(<130 / 80 \mathrm{mmHg}$ en diabéticos) y de colesterol LDL los establecidos para ATP-III para cada grupo de riesgo.

Resultados. Se incluyeron 12.954 pacientes (49,9\% mujeres, 62,1 $\pm 10,7$ años). El 12,6\% pertenecía al grupo de bajo riesgo, el $45 \%$ al de riesgo medio y el $42,4 \%$ al de riesgo alto. El grado de control fue diferente según el grupo de riesgo (p < 0,0001). Así, para la presión arterial se alcanzó en un 37,5\% de los sujetos de riesgo bajo, en un 30,2\% de los del grupo de riesgo medio y en un 15,4\% de los de riesgo alto, y para el colesterol LDL se consiguió en el 65,6\% del grupo de riesgo bajo, el $28 \%$ del de riesgo medio y el $12,3 \%$ del de riesgo alto. Globalmente, si consideramos el control adecuado de presión arterial y de colesterol LDL, sólo el 25,8\% de los de bajo riesgo estaba controlado, el $9,6 \%$ de los de riesgo medio y el $2,7 \%$ de los de riesgo alto.

Conclusiones. La mayoría de los hipertensos asistidos diariamente en Atención Primaria en España son de riesgo medio o alto. Las cifras de control de presión arterial y colesterol LDL en los hipertensos en general son bajas y son muy pocos los pacientes que tienen cifras adecuadas de ambos factores de riesgo. Pero las tasas de control son aún peores según aumenta el riesgo coronario; de hecho, menos del 3\% de los pacientes de alto riesgo tienen bien controlados ambos parámetros, con las implicaciones clínicas que ello conlleva.
\end{abstract}

Objectives and methods. The aim of this cross-sectional and multicenter study was to determine the coronary risk of hypertensive patients attended in Spanish Primary Care and to evaluate whether blood pressure and LDL-cholesterol (LDL-c) control rates could change according to the ATP-III risk groups. Good blood pressure control was considered $<140 / 90 \mathrm{mmHg}(<130 / 80 \mathrm{mmHg}$ for diabetics) and LDL-c according to the established by ATP-III for every risk group.

Results. A total of 12,954 patients were included in the study (49.9\% women, mean age $62.1 \pm 10.7$ years). Of these, $12.6 \%$ belonged to the group of low risk, $45 \%$ to the medium risk group and $42.4 \%$ to the high risk group. The control rates were different according to the risk group ( $\mathrm{p}<0.0001$ ). Blood pressure control: $37.5 \%$ in low risk, 30.2 $\%$ in medium and $15.4 \%$ in high risk group. LDL-c control: $65.6 \%$ in low risk group, $28 \%$ in medium risk group and $12.3 \%$ in high risk group. Only $25.8 \%$ of the patients of low risk were controlled for both blood pressure and LDL-c, $9.6 \%$ of medium risk group and $2.7 \%$ of high risk group.

Conclusions. The majority of hypertensive patients daily attended in Primary Care setting in Spain belongs to the medium or high coronary risk groups. Blood pressure and LDL-c controls rates in hypertensive population are low, and very few patients have both risk factors controlled. The control rates decline when the risk increases. In fact, less than 3\% of high-risk patients have both parameters well controlled, what may result in significant clinical implications.

Palabras clave: hipertensión arterial; lípidos; control; Atención Primaria; España

Key words: hypertension; lipids; control; Primary Care; Spain 


\section{Introducción}

Tanto la hipertensión arterial (HTA) como la hipercolesterolemia constituyen factores de riesgo cuya relación con la enfermedad cardiovascular es bien conocida. El riesgo añadido que supone la HTA en el desarrollo de una patología cardiovascular ha sido reiteradamente señalado por diversos organismos. Tanto en el séptimo informe del Comité Conjunto Americano (JNC-7) ${ }^{1}$ como en las guías europeas y españolas de $\mathrm{HTA}^{2-4}$ se destaca que los niveles de presión arterial (PA) muestran una relación positiva y continua con el riesgo de presentar infarto de miocardio, ictus, insuficiencia cardíaca e insuficiencia renal. Ya en 1990, MacMahon et al demostraron que reducciones en las cifras de PA producían un descenso en la incidencia de infarto cerebral y eventos coronarios ${ }^{5}$. Aunque tradicionalmente el control de la PA se había considerado como el objetivo terapéutico principal ${ }^{6}$, actualmente las principales guías señalan que no se debe limitar únicamente a este aspecto, sino que se debe centrar en el manejo global del riesgo cardiovascular, y abordar, por tanto, el tratamiento de una manera integral ${ }^{1-4}$. Varios estudios han señalado que en la práctica habitual la mayoría de los pacientes hipertensos que acuden diariamente a las consultas de Atención Primaria son de alto riesgo ${ }^{7,8}$. Sin embargo, y aunque la Atención Primaria supone el enclave ideal para la detección y control de los diferentes factores de riesgo, se ha demostrado que todavía presenta importantes carencias en cuanto a la correcta identificación, valoración y manejo del riesgo cardiovascular ${ }^{9-13}$.

Las recomendaciones del tercer informe del National Cholesterol Education Program-Adult Treatment Pannel de Estados Unidos (NCEP-ATP III) ${ }^{14}$ establecen como objetivo prioritario para la prevención primaria y secundaria de la enfermedad coronaria, el control de los niveles de colesterol LDL (c-LDL). Es bien sabido que la prevalencia de la hipercolesterolemia es mayor entre la población hipertensa que entre la normotensa ${ }^{15}$. De hecho, la HTA y la dislipemia manifiestan un efecto sinérgico en el desarrollo de la aterosclerosis, ya que la suma de ambos factores de riesgo produce un incremento en el desarrollo de lesiones vasculares ateromatosas ${ }^{16}$. El enfoque terapéutico del hipertenso debe ser considerado desde un punto de vista global, ya que el tratamiento de los diferentes factores de riesgo asociados puede modificar sustancialmente el pronóstico. Este aspecto quedó bien demostrado en el estudio ASCOT ${ }^{17,18}$, donde se observó que el tratamiento hipolipemiante con atorvastatina $10 \mathrm{mg}$ en sujetos hipertensos y riesgo elevado con un colesterol total $\leq 250 \mathrm{mg} / \mathrm{dl}$ puede disminuir de forma muy significativa $(36 \%)$ la incidencia de eventos cardiovasculares.

Los estudios epidemiológicos que se han realizado en España habitualmente se han centrado en el análisis de los diferentes factores de riesgo cardiovascular por separado, y generalmente no se ha valorado el control de otros factores de riesgo asociados y el problema del riesgo cardiovascular en su conjunto. De hecho, en nuestro país no hay estudios que se hayan enfocado en el control global de la PA y del c-LDL en la población hipertensa. El estudio Prevención cardiovascular en España en Atención Primaria: intervención sobre el colesterol en hipertensión (PRESCOT) es un estudio transversal diseñado para conocer el perfil clínico de la población hipertensa asistida en Atención Primaria en España y su grado de control tensional y lipídico (c-LDL) en la práctica clínica diaria y evaluar si el nivel de control de ambos factores de riesgo varía según el riesgo coronario (clasificación NCEP-ATP III) de los pacientes.

\section{Pacientes y métodos}

\section{Población a estudio}

Para la realización del presente estudio se contó con la participación de aproximadamente 2.000 investigadores de centros de Atención Primaria, distribuidos por todo el territorio nacional de manera aleatoria para así aumentar la representatividad de los datos obtenidos. El estudio se realizó en el primer trimestre de 2004. Cada investigador debía incluir por muestreo consecutivo un promedio de seis pacientes que acudieran a la consulta y cumplieran los siguientes criterios: mayores de 18 años, ambos sexos, hipertensos (con PA sistólica mayor de $140 \mathrm{mmHg}$ y/o diastólica mayor de $90 \mathrm{mmHg}$ o historia de HTA en tratamiento antihipertensivo) y que pudieran aportar una analítica, con un estudio lipídico completo, con una antiguiedad no superior a seis meses. Para que el estudio tuviera una distribución amplia y fuera representativo de la población que acude diariamente a los centros de Atención Primaria, no hubo criterios de exclusión específicos. 


\section{Metodología y recogida de datos}

Para la medida de la PA, el paciente debía estar cinco minutos en reposo. Posteriormente el investigador realizó dos medidas y tomó la media de estas dos determinaciones. Se consideró que el paciente hipertenso presentaba un control adecuado de PA si cumplía los criterios de las guías europeas (ESH-ESC 2003) $)^{2}$ : PA inferior a 140 y $90 \mathrm{mmHg}$ o inferior a 130 y $80 \mathrm{mmHg}$ en caso de diabetes. Se clasificaron a los pacientes como dislipémicos si presentaban diagnóstico establecido de alteración del perfil lipídico (hipercolesterolemia, hipertrigliceridemia, hiperlipemia mixta) o tratamiento hipolipemiante. Para evaluar el control de c-LDL se utilizaron los objetivos terapéuticos establecidos según los grupos de riesgo coronario que establece el informe del NCEP-ATP III ${ }^{14}$, es decir, un c-LDL menor de $100 \mathrm{mg} / \mathrm{dl}$ en los pacientes de riesgo alto, menor de $130 \mathrm{mg} / \mathrm{dl}$ en los de riesgo intermedio y menor de160 mg/dl en los de riesgo bajo. La medición del perímetro abdominal se hizo en el punto medio entre la cresta iliaca y el reborde costal, considerándose patológico un perímetro de cintura $\geq 102 \mathrm{~cm}$ en el varón $\mathrm{y} \geq 88 \mathrm{~cm}$ en la mujer. Se definió el sedentarismo como la realización de una actividad física menor a 30 minutos de paseo diario de intensidad moderada durante al menos 4 días y el enolismo como un consumo diario de alcohol superior a tres unidades de bebida ( 1 unidad de bebida $=1$ cerveza o 1 copa de vino o $1 / 2$ copa de licor). Se consideró insuficiencia renal una creatinina sérica $>1,5 \mathrm{mg} / \mathrm{dl}$ en varones y > $1,4 \mathrm{mg} / \mathrm{dl}$ en mujeres, como establecen las guías europeas ${ }^{2}$. Siguiendo estas mismas recomendaciones se definió proteinuria como un cociente albúmina/creatinina $>300 \mathrm{mg} / \mathrm{g}$ y microalbuminuria como un cociente albúmina/creatinina entre $30-299 \mathrm{mg} / \mathrm{g}$. La presencia de hipertrofia ventricular izquierda se consideró según la información suministrada por el investigador, que pudo establecer el diagnóstico por electrocardiograma y/o ecocardiograma. Los diagnósticos de insuficiencia cardíaca y de arteriopatía periférica se incluyeron según los datos aportados por cada investigador.

Para el cálculo del riesgo de enfermedad coronaria en esta población se siguió la estratificación en grupos de riesgo establecida por la NCEP-ATP III, puesto que es la clasificación que mejor define los objetivos del c-LDL según el nivel de riesgo ${ }^{14}$. Se consideraron pacientes de riesgo coronario alto aquéllos con enfermedad coronaria o equivalente (riesgo de presentar un evento coronario a 10 años > $20 \%$ ); de riesgo medio, aquellos pacientes con dos o más factores de riesgo (riesgo coronario a 10 años $\leq$ $20 \%$ ) y de riesgo bajo, aquéllos con uno o ningún factor de riesgo cardiovascular. La NCEP-ATP III define como factores de riesgo el hábito tabáquico, la HTA, niveles disminuidos de colesterol HDL $(<40$ $\mathrm{mg} / \mathrm{dl}$ ), historia familiar de enfermedad coronaria prematura (en familiares de primer grado $<55$ años si es varón y $<65$ años si es mujer) y la edad ( $\geq 45$ años en el varón y $\geq 55$ años en la mujer). Como equivalente coronario incluye la arteriopatía periférica, el aneurisma de aorta abdominal, la enfermedad carotídea y la diabetes. Para el diagnóstico de síndrome metabólico se utilizaron los criterios NCEP-ATP III $^{14}$. Un paciente cumplía criterios para síndrome metabólico si presentaba tres o más de los siguientes factores de riesgo: obesidad central (perímetro de cintura $>102 / 88 \mathrm{~cm}$; varones/mujeres); triglicéridos $\geq$ $150 \mathrm{mg} / \mathrm{dl}$; colesterol-HDL $<40 / 50 \mathrm{mg} / \mathrm{dl}$ (varones/mujeres); glucosa en ayunas $\geq 110 \mathrm{mg} / \mathrm{dl}$ y PA $\geq$ 130/85 mmHg. Se excluyeron del análisis final a aquellos sujetos cuyos datos no estuviesen adecuadamente recogidos de forma que resultasen incoherentes y/o incompletos.

\section{Análisis estadístico}

Las pruebas estadísticas se realizaron dependiendo de la naturaleza de las variables. El estudio de la relación de variables categóricas se realizó mediante el test Chi-Cuadrado (en el caso de que más del 20\% de las celdas tuvieran una frecuencia esperada menor a 5 se utilizó el test exacto de Fisher). La comparación de variables continuas entre grupos de pacientes se realizó mediante el test de la «t» de Student. Para la determinación de los factores predictores de mal control de la PA, se realizó un análisis multivariante mediante regresión logística de aquellos factores que pudieran influir en el grado de control de la PA. Las variables incluidas en el análisis de regresión logística fueron datos demográficos, factores de riesgo cardiovascular y patologías asociadas, tratamientos antihipertensivos, hipolipemiantes y antitrombóticos, así como datos analíticos. El diseño de la base de datos estuvo sometido a reglas de coherencia internas y rangos para controlar las incoherencias y/o incorrecciones en la recogida y la tabulación de los datos (SPSS versión 11.0.1, Data Entry). 


\section{Resultados}

En el estudio PRESCOT se incluyeron un total de 15.707 pacientes. Del total de pacientes incluidos, finalmente se analizaron $12.954(82,5 \%)$. Se depuró de forma muy estricta la base de datos excluyendo aquellos sujetos que no cumplían los criterios de selección y/o el cuaderno de recogida de datos resultaba incompleto y/o incoherente. Por lo tanto, para los análisis correspondientes se consideraron únicamente los casos que estaban correctamente documentados y aportaban la información necesaria, para poder así asegurar al máximo la validez de los parámetros objeto de estudio, aunque ello pudiera ir en detrimento del tamaño muestral. La población final del estudio $(n=12.954)$ estuvo compuesta en un $49,9 \%$ por mujeres $(n=6.486)$, la edad media fue de 62,1 $\pm 10,7$ años, el índice de masa corporal (IMC) de 28,5 \pm $4,1 \mathrm{~kg} / \mathrm{m}^{2}$, el perímetro de cintura fue $101,8 \pm 14,3 \mathrm{~cm}$ en varones y $96,9 \pm 17,1$ en mujeres, y la PA media fue de 144,1 $\pm 14,2 / 85 \pm 9,1 \mathrm{mmHg}$. La edad media en el diagnóstico de HTA fue de 55,6 \pm 9,8 años y el tiempo medio de evolución de la HTA de 6,7 $\pm 5,4$ años. En la tabla 1 se resumen las características de la muestra. Cabe destacar que en esta población de pacientes hipertensos, el 76\% presentaba además dislipemia, el 49,8\% síndrome metabólico y casi un 30\% diabetes mellitus. Asimismo, el $32,1 \%$ presentaba afectación cardíaca (hipertrofia ventricular izquierda, cardiopatía isquémica e insuficiencia cardíaca), el 10,5\% afectación renal (microalbuminuria, proteinuria e insuficiencia renal), el $5,4 \%$ enfermedad vascular cerebral y el 4,4\% arteriopatía periférica. En cuanto al tratamiento farmacológico de la HTA, únicamente el 5,5\% de los pacientes estaba sin medicación. De los pacientes tratados con antihipertensivos, el 55,9\% sólo estaba tomando un fármaco mientras que el 38,6\% tomaba varios (el 29,8\% dos fármacos y el 8,8\% tres o más). En la tabla 2 se desglosan los tipos de medicación que tomaban dichos pacientes. La clase farmacológica más frecuentemente utilizada en monoterapia fue la de inhibidores de la enzima convertidora de angiotensina (IECA) y las combinaciones más frecuentes fueron las de un diurético con algún inhibidor del sistema renina-angiotensina aldosterona (SRA), principalmente la combinación de IECA y diurético. Si analizamos los tipos de tratamiento antihipertensivo de forma global, incluyendo mono y politerapia (fig. 1), los fármacos más prescritos fueron los IECA seguidos de los diuréticos y de los antagonistas de los receptores de angiotensina II (ARA II). En la fig. 2 se resumen otros tratamientos concomitantes que tomaban los sujetos de la muestra. Aunque el $76 \%$ de los pacientes presentaba dislipemia, sólo el $41 \%$ del total estaba bajo tratamiento hipolipemiante. 
Tabla 1. Características basales de la muestra $(n=12.954)$

Datos biodemográficos $(n=12.954)$

Sexo (V/M)

Edad (años)

IMC $\left(\mathrm{kg} / \mathrm{m}^{2}\right)$

Perímetro de cintura $(\mathrm{cm})$

Factores de riesgo

Dislipemia

Diabetes

Hábito tabáquico

Con antecedentes familiares de enfermedad cardiovascular

Enfermedad cardiovascular

Afectación cardíaca

Hipertrofia ventricular izquierda

Cardiopatía isquémica

Insuficiencia cardíaca

Afectación renal

Microalbuminuria

Proteinuria

Insuficiencia renal.

Creat $>1,5 / 1,4 \mathrm{mg} / \mathrm{dl}$ (V/M)

Enfermedad vascular cerebral

Arteriopatía periférica

Datos clínicos

PAS (mmHg)

PAD $(\mathrm{mmHg})$

Perfil bioquímico

Colesterol total (mg/dl)

Triglicéridos $(\mathrm{mg} / \mathrm{dl})$

Colesterol LDL (mg/dl)

Colesterol HDL (mg/dl)

Glucemia (mg/dl)

Ácido úrico $(\mathrm{mg} / \mathrm{dl})$
V: $50,1 \%(\mathrm{n}=6.486)$

M: $49,9 \%(n=6.468)$

$$
62,1 \pm 10,7
$$

$\mathrm{V}: 28,4 \pm 3,6 ; \mathrm{M}: 28,5 \pm 4,6$

$\mathrm{V}: 101,8 \pm 14,3 ; \mathrm{M}: 96,9 \pm 17,1$

$76,0 \%(\mathrm{n}=9.850)$

$29,9 \%(\mathrm{n}=3.868)$

$37,6 \%(\mathrm{n}=4.875)$

$32,5 \%(n=4.209)$

$32,1 \%(\mathrm{n}=4.164)$

$11,9 \%(\mathrm{n}=1.539)$

$13,6 \%(\mathrm{n}=1.765)$

$4,6 \%(n=591)$

$10,5 \%(\mathrm{n}=1.358)$

$6,6 \%(\mathrm{n}=851)$

$1,1 \%(\mathrm{n}=143)$

$2,7 \%(\mathrm{n}=351)$

$5,4 \%(\mathrm{n}=699)$

$4,4 \%(\mathrm{n}=566)$

$144,1 \pm 14,2$

$85,0 \pm 9,1$

$233,1 \pm 41,8$

$158,1 \pm 80,0$

$150,9 \pm 92,8$

$53,3 \pm 18,1$

$110,5 \pm 32,0$

$5,4 \pm 1,9$

Porcentajes calculados sobre el total de pacientes valorables $(\mathrm{n}=12.954)$. Los pacientes pudieron presentar más de una patología de riesgo simultáneamente.

IMC: índice de masa corporal; M: mujer; PAD: presión arterial diastólica; PAS: presión arterial sistólica; V: varón

Tabla 2. Tipos de tratamiento antihipertensivo (con combinaciones)

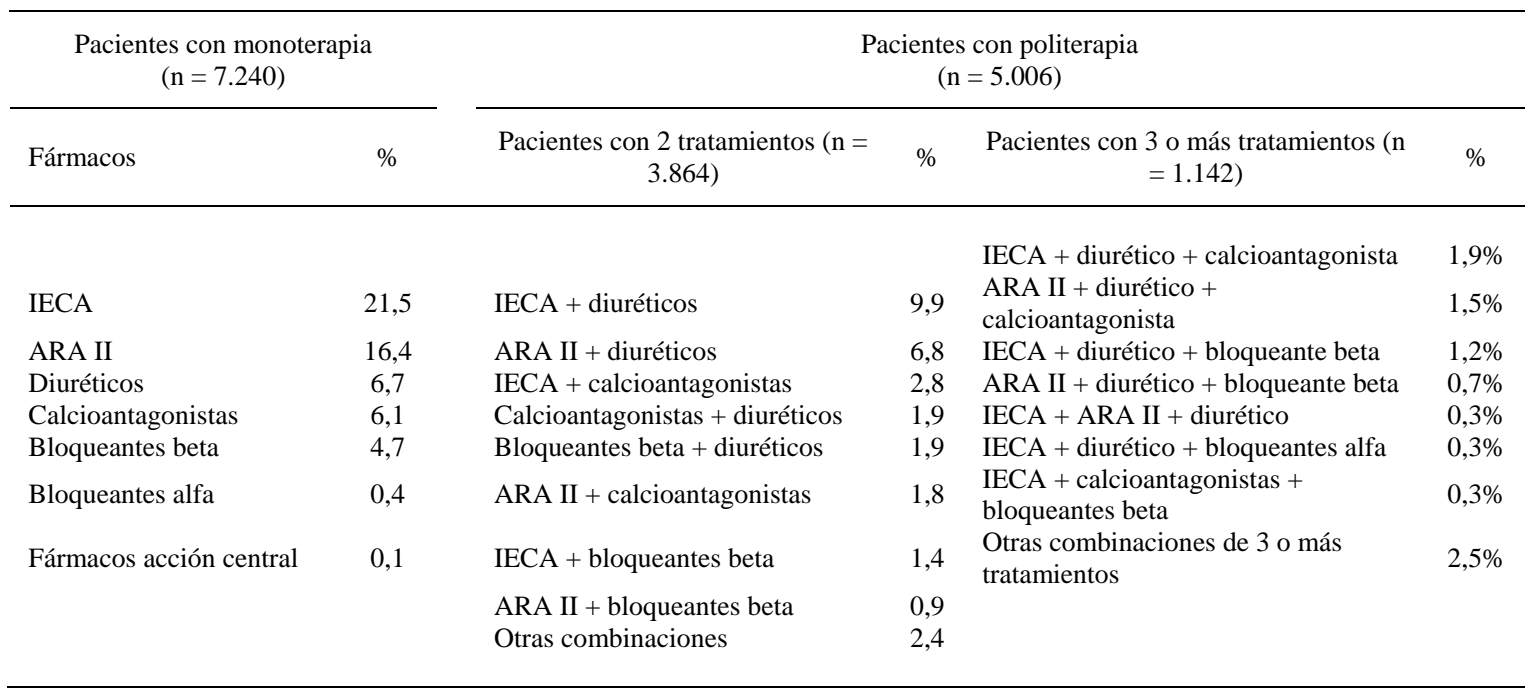

ARA II: antagonistas de los receptores de angiotensina II; IECA: inhibidores de la enzima convertidora de angiotensina. 


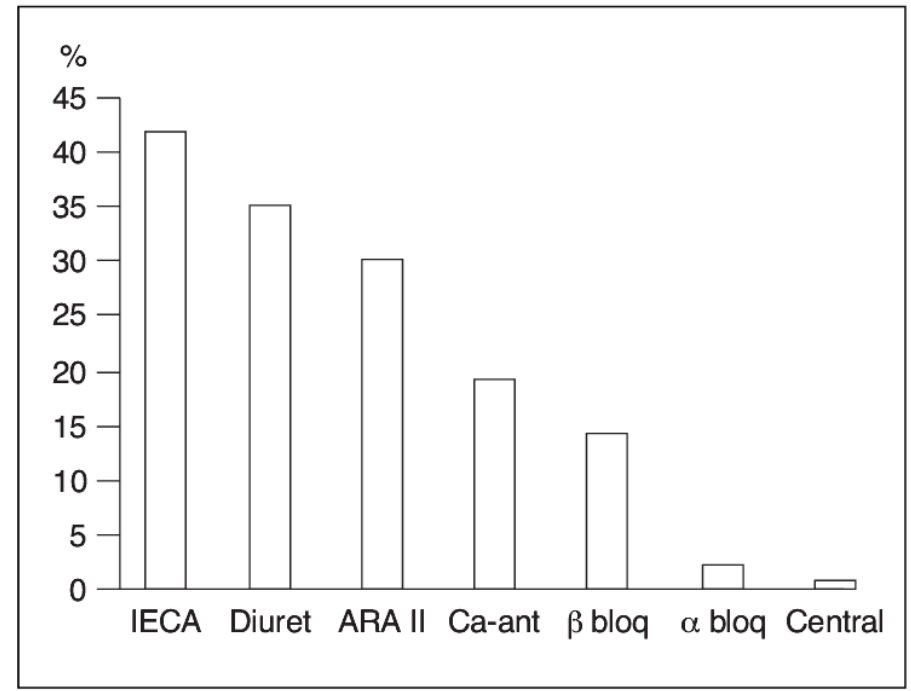

Fig. 1. Tipos de tratamiento antihipertensivo de forma global.

IECA: inhibidores de la enzima convertidora de angiotensina; Diuret: diuréticos; ARA II: antagonistas de los receptores de angiotensina II; Ca-ant: calcioantagonistas; $\beta$-bloq: bloqueantes beta; $\alpha$-bloq: bloqueantes alfa; central: fármacos de acción central

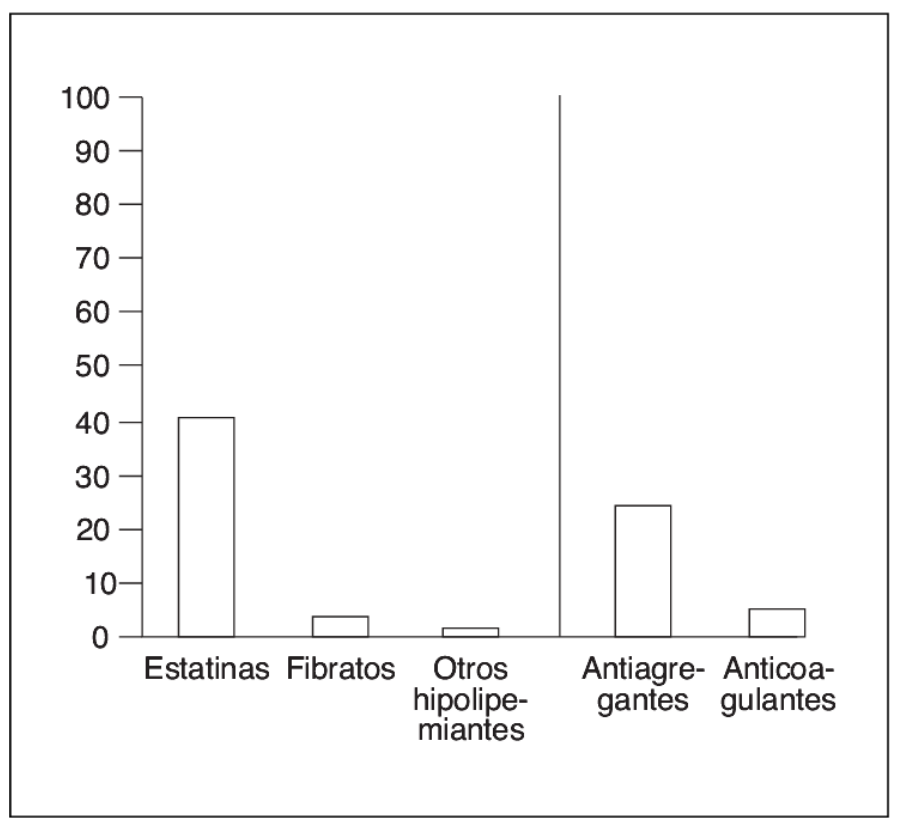

Fig. 2. Otros tratamientos concomitantes.

En la fig. 3 se señala la distribución de la muestra según el grupo de riesgo de enfermedad coronaria. En cuanto a las características clínicas de los pacientes según los diferentes grupos de riesgo, se observó que los de alto riesgo tenían significativamente mayor edad $(64,5 \pm 10$ años frente a 60,6 $\pm 10,5$ en los de riesgo medio y 59,6 $\pm 12,4$ en los de riesgo bajo, $\mathrm{p}<0,0001)$, mayor IMC $\left(29,1 \pm 4,2 \mathrm{~kg} / \mathrm{m}^{2}\right.$ frente a 28,1 $\pm 3,9$ y 27,6 $\pm 4,2, \mathrm{p}<0,0001)$ y mayor PA sistólica $(144,6 \pm 14,7 \mathrm{mmHg}$ frente a 144,3 $\pm 13,6$ y $142,0 \pm$ $14,4, \mathrm{p}<0,0001)$. Por otra parte, en el grupo de riesgo bajo había más mujeres $(79,1 \%$ frente al $44,9 \%$ en el de riesgo medio y $46,9 \%$ en el de riesgo alto, $\mathrm{p}<0,0001)$. 


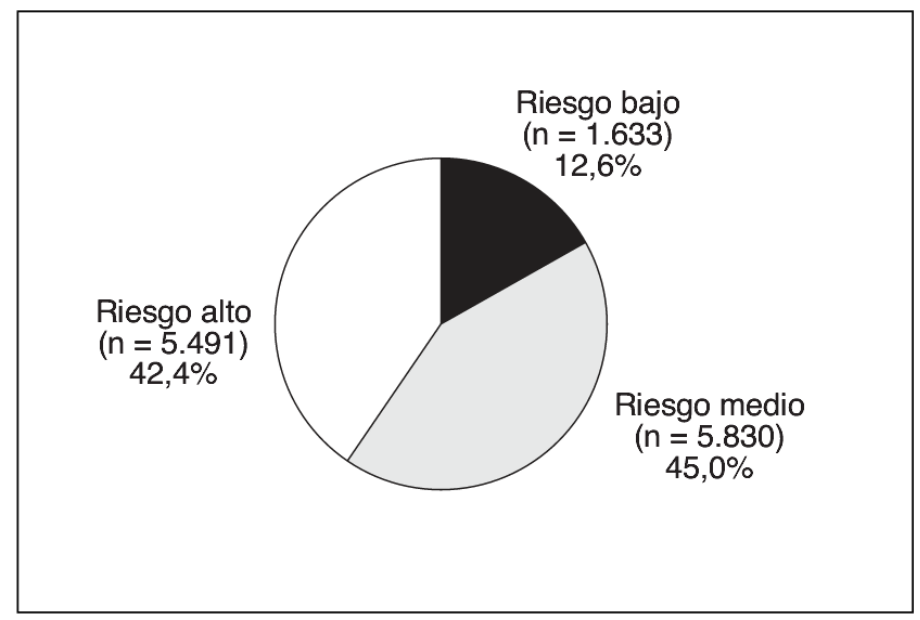

Fig. 3. Clasificación de los pacientes según el riesgo de enfermedad coronaria

En cuanto al control de la PA, únicamente el 24,8\% tenía bien controlada la PA sistólica (PAS) y diastólica (PAD), el 28,5\% la PAS y un 50,9\% la PAD. Se realizó un análisis de regresión logística y se analizaron las características mostradas por los pacientes hipertensos (datos demográficos, factores de riesgo, patologías, tratamientos antihipertensivos, hipolipemiantes, antitrombóticos y datos analíticos) que pudieran influir en el control de la PA. Las variables que mostraron significación respecto al mal control fueron la presencia de diabetes, la afectación renal, el hábito enólico, el sedentarismo y la presencia de hipertrofia ventricular izquierda (tabla 3). Respecto al grado de control del c-LDL, únicamente el $26 \%$ cumplió con los objetivos terapéuticos. Si analizamos conjuntamente el grado de control de la PA y del cLDL, sólo el 8,6\% de los pacientes tenían ambos parámetros bien controlados (fig. 4). Asimismo, en la figura 4 se señala el grado de control de la PA, del c-LDL y de ambos según el grupo de riesgo. Como se puede observar, el grado de control en todos los parámetros fue siempre peor cuanto mayor era el riesgo coronario, por lo que se llegó a tener cifras adecuadas de ambos parámetros sólo en el 2,7\% de los pacientes en el grupo de alto riesgo. Si analizamos a dos subgrupos con riesgo coronario elevado como son los diabéticos y los que presentan síndrome metabólico, observamos la misma tendencia. El porcentaje de pacientes bien controlados de PA, c-LDL y ambos (PA y c-LDL) fue de 6,3\%, 12\% y 1\%, respectivamente en los hipertensos diabéticos frente a 32,7\%,31,9\% y 11,9\% en hipertensos sin diabetes, $\mathrm{p}<0,0001, \mathrm{y} 13,8 \%, 16,9 \%$, y $3,7 \%$ respectivamente en hipertensos con síndrome metabólico frente a $35,6 \%, 34,7 \%$ y $13,6 \%$ en hipertensos sin síndrome metabólico, $\mathrm{p}<0,0001$.

Tabla 3. Variables que en el análisis de regresión logística se asociaron a un mal control de la presión arterial $(\mathrm{p}<0,05)$

\begin{tabular}{lcc}
\hline Variables & OR & Intervalo de confianza 95\% \\
\hline & & $5,51-7,37$ \\
Diabetes & 6,37 & $2,16-3,55$ \\
Afectación renal & 2,77 & $1,27-1,56$ \\
Hábito enólico & 1,41 & $1,23-1,47$ \\
Sedentarismo & 1,35 & $1,06-1,42$ \\
Hipertrofia ventricular izquierda & 1,23 & \\
\hline
\end{tabular}




\section{Discusión}

En la actualidad existe una mayor conciencia del problema que supone la detección y el adecuado control de la HTA en nuestro país. De hecho, en los últimos años se han desarrollado diversos estudios epidemiológicos que analizan el control de la PA y que han observado una mejora constante en las últimas décadas $^{9-13,19}$. Sin embargo, cada vez resulta más evidente que la única forma de reducir el riesgo cardiovascular en un hipertenso es mediante un tratamiento integral dirigido no sólo a los valores de la PA, sino al conjunto de factores de riesgo y lesiones orgánicas subclínicas ${ }^{20}$. Por otra parte, los objetivos de control de factores de riesgo modificables como la PA y el c-LDL deben ser diferentes según el riesgo del paciente, de forma que sean más exigentes cuanto mayor sea el riesgo cardiovascular. Si bien, de forma global, España presenta un menor riesgo coronario que otros países europeos ${ }^{21}$, en la práctica clínica los pacientes que acuden diariamente a la consulta de Atención Primaria son cada vez de mayor riesgo. De hecho, en este estudio muy pocos pacientes eran de bajo riesgo. Resulta de especial relevancia el hecho de que, en nuestra serie, había una proporción muy elevada de factores de riesgo asociados, pero principalmente hay que tener presente que casi un tercio presentaba afectación cardíaca, lo que confirma el riesgo elevado de esta población. Estos hallazgos son concordantes con otros estudios previos realizados en Atención Primaria en nuestro país en los últimos años ${ }^{7,8,22}$. Como es lógico, en las unidades hospitalarias especializadas de HTA los pacientes presentan un riesgo todavía mayor ${ }^{23}$. Así, en el CLUE, el $78 \%$ de los pacientes eran de riesgo alto o muy alto ${ }^{24}$.

Por tanto, en la práctica clínica diaria la población hipertensa habitualmente es de riesgo alto, lo que obliga a un control estricto de los diferentes factores de riesgo y alcanzar los niveles adecuados de control debe ser un objetivo prioritario en estos pacientes. Pero para ello es fundamental reconocer el riesgo cardiovascular. El estudio DIORISC demostró que frecuentemente el médico de Atención Primaria infraestima el riesgo de los pacientes ${ }^{8}$, lo que dificulta aún más lograr los objetivos propuestos. Los resultados del estudio PRESCOT demuestran que cuanto mayor es el riesgo, peor es el control tanto de la PA como del c-LDL, lo que puede tener una influencia decisiva en el pronóstico, ya que son los pacientes de alto riesgo los que más se favorecen de la intervención terapéutica. En consecuencia, hay que considerar que entre los factores que intervienen en el control de factores de riesgo en la práctica clínica, está no sólo la dificultad de alcanzar unos objetivos cada vez más estrictos según aumenta el riesgo, sino también la infraestimación o la falta de reconocimiento del riesgo cardiovascular por parte de los médicos.

En el grado de control de la PA, nuestros resultados indican que menos de un cuarto de los pacientes tiene un control adecuado y que éste empeora según se incrementa el riesgo coronario. En el estudio PRESCAP $^{9}$ se analizó el grado de control de la PA en una muestra de hipertensos atendidos en Atención Primaria, y determinaron que algo más de un tercio presentaba un buen control tensional. Mismo objetivo tuvo otro estudio, el CONTROLPRES $2003^{12}$, en donde el 38,8\% de los sujetos alcanzó cifras adecuadas de PA. En ambos casos, el control de la PAS fue menor que el de la PAD. El estudio PREVENCAT ${ }^{13}$ estudió a pacientes con HTA, diabetes y/o hipercolesterolemia. El $40 \%$ de los pacientes tenía un adecuado control de la PA y un $42,6 \%$ cifras normales de colesterol. El estudio CARDIOTENS ${ }^{25}$ analizó el grado de control de la HTA asociada con enfermedades cardiovasculares. Un tercio de la muestra eran hipertensos y el 19\% presentaba antecedentes de cardiopatía. Menos de una quinta parte de los hipertensos con cardiopatía estaban adecuadamente controlados. Estos resultados confirman no sólo que el control de la PA está lejos de las recomendaciones actuales, sino que además la situación es todavía peor en aquellos sujetos en los que el control debería ser más estricto ${ }^{24}$. Nuestro estudio, realizado en una muestra muy amplia y representativa de la población hipertensa $(n=12.954)$ corrobora también este hecho. Pero el mal control de la PA en los hipertensos no es un problema exclusivo de Atención Primaria. En el estudio CLUE menos de la mitad de los pacientes estaban adecuadamente controlados, y en la población de alto riesgo este porcentaje bajaba hasta el $18 \%$ de los pacientes ${ }^{24}$. Además de los factores ya reseñados como la infraestimación del riesgo y las dificultades de lograr objetivos de control muy rigurosos, existen otros factores que pueden intervenir también en el mal control de la PA, como la poca relevancia que se sigue dando al control adecuado de la $\mathrm{PAS}^{11}$ y la inercia clínica, que lleva a introducir escasos cambios en el tratamiento antihipertensivo del hipertenso no controlado ${ }^{20,26}$.

En cuanto al control del c-LDL, los resultados del estudio PRESCOT reflejan un preocupante escaso control de los lípidos. Al igual que ocurre con la PA, este control disminuye aún más cuanto mayor es el riesgo global del paciente y si analizamos en nuestra serie el porcentaje con un adecuado control de la PA y del c-LDL, sólo logran el objetivo de un control adecuado conjunto el 8,6\%. Es de vital importancia el adecuado control de los distintos factores de riesgo cardiovascular, ya que es la única forma de reducir el riesgo cardiovascular global de los pacientes. De hecho, el estudio ASCOT constituye un magnífico ejemplo de las ventajas del tratamiento integral en la HTA de alto riesgo ${ }^{17,18}$. Pero la relación entre HTA y dislipemia es una interrelación bidireccional. Así, el estudio LIPYCARE ${ }^{27}$, que analizó a pacientes con hipercolesterolemia en el ámbito de la Atención Primaria, observó la presencia muy frecuente de otros 
factores de riesgo asociados a la dislipemia, siendo el más frecuente la HTA (45,1\%). Además, aproximadamente la mitad de los sujetos eran de riesgo alto o muy alto. Al igual que en este estudio, en nuestra muestra se demostró que existe una elevada prevalencia de dislipemia en los hipertensos, lo que sin duda incrementa el riesgo y aumenta las exigencias de control de la PA y el c-LDL.

Varios estudios han analizado la situación del control lipídico en la población española. El estudio REALITY $^{28}$ y el ELIPSE ${ }^{29}$, que analizaron pacientes de alto riesgo coronario en el ámbito de la Atención Primaria, objetivaron que aproximadamente una décima parte de los mismos tenían cifras adecuadas de cLDL. En nuestra serie, casi la mitad de los sujetos toma algún tipo de hipolipemiante (sobre todo estatinas), con un escaso control de los niveles de c-LDL, que empeora según aumenta el riesgo coronario. El estudio Barbanza ${ }^{30}$ también incluyó a pacientes coronarios en Atención Primaria, con resultados parecidos (menos de la mitad de los pacientes recibía tratamiento hipolipemiante con un control adecuado en los niveles de c-LDL de tan sólo el 10\%). Similares resultados se han encontrado en el ámbito hospitalario ${ }^{31,32}$. En un reciente estudio americano ${ }^{33}$, que incluyó a hipertensos que iniciaban tratamiento antihipertensivo y que además presentaban enfermedad coronaria previa, se observó que sólo el $11,5 \%$ de los mismos ya estaba tomando estatinas en el momento de la inclusión, y que menos de un tercio inició tratamiento con hipolipemiantes durante el primer año de seguimiento. Es decir, más de la mitad de los pacientes no recibió tratamiento con hipolipemiantes, a pesar del beneficio que ha mostrado el uso de estatinas.

Hay que señalar que por el propio diseño del estudio, de corte transversal, el presente trabajo tiene algunas limitaciones metodológicas que conviene recordar. Aunque la selección de pacientes se realizó de manera consecutiva según la práctica diaria, esta selección no fue aleatoria, por lo que puede ocurrir que los resultados no sean exactamente extrapolables a la población hipertensa en general. Sin embargo, el alto número de pacientes incluidos y la meticulosidad en la obtención y control de los datos analizados hacen que la población incluida represente con cierta fiabilidad una instantánea de la población hipertensa que acude a diario a las consultas de Atención Primaria en España.

En conclusión, el estudio PRESCOT, a diferencia de otros trabajos realizados en Atención Primaria, analiza al paciente hipertenso valorando el riesgo coronario global, no sólo el control de la PA sino también el de otros factores de riesgo como el c-LDL en una muestra muy amplia y representativa de la población hipertensa que acude a diario a las consultas de Atención Primaria y, por lo tanto, aporta una visión más completa del paciente en su conjunto y en consecuencia una valoración del mismo más adecuada. Nuestros resultados indican que la mayoría de los hipertensos que se ven en Atención Primaria padecen ya HTA de riesgo coronario moderado o alto, y presentan un pobre control de la PA y del cLDL, sobre todo cuanto mayor es el riesgo coronario, lo que llega hasta al 2,7\% de control conjunto en los pacientes de riesgo más alto. Estos resultados obligan a realizar una profunda reflexión sobre la realidad del manejo de los hipertensos de alto riesgo y hace pensar que se debe optimizar el tratamiento de estos pacientes utilizando mejor los recursos terapéuticos disponibles en la actualidad para mejorar este preocupante panorama. Se debe insistir tanto en las medidas no farmacológicas (dieta y ejercicio), que sin duda van a ayudar a un mejor control de la PA y del c-LDL, como en la adecuada utilización de los diferentes recursos para optimizar el tratamiento farmacológico (utilización de combinaciones fijas en HTA, mayor uso de hipolipemiantes en la población hipertensa, uso de determinados fármacos preferibles para algunas situaciones clínicas...), así como intentar evitar la inercia clínica.

\section{Agradecimientos}

Los autores quieren expresar su más profundo y sincero agradecimiento a todos los médicos de Atención Primaria que han participado con todo entusiasmo y dedicación en el estudio PRESCOT. Es evidente que sin su inestimable ayuda y la calidad de su trabajo esta publicación no hubiera sido posible. Desafortunadamente la larga lista de investigadores que intervinieron hace imposible transcribirla al completo. También queremos agradecer expresamente a los laboratorios Almirall-Prodesfarma y a Biométrica la financiación y el apoyo estadístico respectivamente, imprescindibles para poder realizar este estudio. 


\section{Bibliografía}

1. A.V. Chobanian, G.L. Bakris, H.R. Black, W.C. Cushman, L.A. Green, J.L. Izzo, and the National High Blood Pressure Education Program Coordinating Committee, et al. The Seventh Report of the Joint National Committee: on prevention, detection, evaluation, an treatment of high blood presure. JAMA, 289 (2003), pp. $2560-2572$.

2. 2003 European Society of Hypertension - European Society of Cardiology guidelines for the management of arterial hypertension. J Hypertens. 2003;21:1011-53.

3. J.R. González-Juanatey, P. Mazón, F. Soria, V. Barrios, L. Rodríguez-Padial, V. Bertomeu. Actualización (2003) de las guías de práctica clínica de la Sociedad Española de Cardiología en hipertensión arterial. Rev Esp Cardiol, 56 (2003), pp. 487-497.

4. R. Marín, P. Armario, J.R. Banegas, C. Campo, A. de la Sierra, M. Gorostidi, et al. Guía española de hipertensión arterial 2005. Hipertension, 22 (Supl 2) (2005), pp. S1-S83.

5. S. MacMahon, R. Peto, J. Cutler, R. Collins, P. Sorlie, J. Neaton, et al. Blood pressure, stroke, and coronary heart disease. Part 1, Prolonged differences in blood pressure: prospective observational studies corrected for the regression dilution bias. Lancet, 335 (1990), pp. 765-774.

6. S. Julius. Five decades of antihypertensive treatment: the unresolved issues. J Hypertens, 18 (Suppl) (2000), pp. S3-S7.

7. A. Sierra, L.M. Ruilope, A. Coca, M. Luque-Otero, en representación del GEDEC (Grupo Español de Estudios Cardiovasculares). Relación entre el perfil de riesgo cardiovascular y la selección y utilización de los fármacos antihipertensivos. Med Clin, 115 (2000), pp. 41-45.

8. V. Barrios, R. Campuzano, G. Peña, G. Guzmán, L.M. Ruilope. Estratificación del riesgo cardiovascular en hipertensión en atención primaria e impacto sobre el tratamiento antihipertensivo. Estudio DIORISC. Hipertensión, 19 (2002), pp. 114-120.

9. J.L. Listerri, G.C. Rodríguez, F.J. Alonso, S. Lou, J.A. División, J.A. Santos, et al. Control de la presión arterial en la población hipertensa española atendida en atención primaria. Estudio PRESCAP 2002. Med Clin (Barc), 122 (2004), pp. 165-171.

10. A. Coca. Evolución del control de la hipertensión arterial en España. Resultados del estudio Controlpres 98. Hipertensión, 15 (1998), pp. 298-307.

11. A. Coca. Evolución del control de la hipertensión arterial en España. Resultados del estudio Controlpres 2001. Hipertensión, 19 (2002), pp. 390-399.

12. A. Coca. Evolución del control de la hipertensión arterial en Atención Primaria en España. Resultados del estudio Controlpres 2003. Hipertensión, 22 (2005), pp. 5-14.

13. L.A. Álvarez-Sala, C. Suárez, T. Mantilla, J. Franch, L.M. Ruilope, J.R. Banegas, et al. Estudio PREVENCAT: control del riesgo cardiovascular en atención primaria. Med Clin (Barc), 124 (2005), pp. 406-410.

14. National Cholesterol Education Program (NCEP) Expert Panel on Detection. Evaluation, and Treatment of High Blood Cholesterol in Adults (Adult Treatment Panel III). Third Report of the National Cholesterol Education Program (NCEP) Expert Panel on Detection, Evaluation, and Treatment of High Blood Cholesterol in Adults (Adult Treatment Panel III) final report. Circulation, 106 (2002), pp. 3143-3221.

15. P. Cía, P. Armario, L. Badimón, J. Redón, Sociedad Española de Arteriosclerosis (SEA) y S.ELELHA. Documentos de consenso Hipertensión arterial en el paciente dislipémico. Clin Invest Arterioscler, 5 (2001), pp. 179-194.

16. R. Velayos, C. Suárez. Hipertensión y dislipidemia. Hipertensión, 9 (2001), pp. 418-428

17. P. Sever, B. Dahlöf, N. Poulter, H. Wedel, G. Beevers, M. Caulfield, et al. For the ASCOT investigators Prevention of coronary and stroke events with atorvastatin in hypertensive patients who have average or lowerthan-average cholesterol concentrations, in the Anglo-Scandinavian Cardiac Outcomes Trial-Lipid Lowering Arm (ASCOT-LLA): a multicentre randomised controlled trial. Lancet, 361 (2003), pp. 1149-1158.

18. B. Dahlof, P.S. Sever, N.R. Poulter, H. Wedel, D.G. Beevers, M. Caulfield, et al. Prevention of cardiovascular events with an antihypertensive regimen of amlodipine adding perindopril as required versus atenolol adding bendroflumethiazide as required, in the Anglo-Scandinavian Cardiac Outcomes TrialBlood Pressure Lowering Arm (ASCOT-BPLA): a multicentre randomised controlled trial. Lancet, 366 (2005), pp. 895-906.

19. B. Gil. Algunas reflexiones y hallazgos de una Unidad de Hipertensión en España. Rev Esp Cardiol, 206 (2006), pp. 144-146.

20. J.R. Banegas. Epidemiología de la hipertensión arterial en España. Situación actual y perspectivas. Hipertensión, 22 (2005), pp. 353-362.

21. R.M. Conroy, K. Pyorala, A.P. Fitzgerald, S. Sans, A. Menotti, G. De Backer, SCORE project group, et al. Estimation of ten-year risk of fatal cardiovascular disease in Europe: the SCORE project. Eur Heart J, 24 (2003), pp. 987-1003.

22. V. Barrios, M. de la Figuera, R. Gabriel, R. Hernández, A. Calderón, L.M. Ruilope, et al. Treatment of high risk hypertensive patients in daily clinical practice. The TARVEST study. J Hypertens, 23 (2) (2005), p. S83.

23. P. García-Pavía, J. González, R. Pastorín, M. Bruna, J. Ráez, A.I. Tabueca, et al. Análisis de las áreas de mejora del control de la hipertensión arterial en Atención Primaria. Rev Esp Cardiol, 206 (2006), pp. 220-224.

24. J.R. Banegas, J. Segura, L.M. Ruilope, M. Luque, R. García-Robles, C. Campo, et al. Blood pressure control and physician management of hypertension in hospital hypertension units in Spain. Hypertension, 43 (2004), pp. $1338-1344$.

25. J.R. González, E. Alegría, J.V. Lozano, J.L. Llisterri, J.M. García, I. González. Impacto de la hipertensión en las cardiopatías en España. Estudio CARDIOTENS 1999. Rev Esp Cardiol, 54 (2001), pp. 1398-2149. 
26. J.L. Llisterri, G.C. Rodríguez, F.J. Alonso, S. Lou, J.A. Divison, J.A. Santos, et al. Blood pressure control in Spanish hypertensive patients in Primary Health Care Centres. PRESCAP 2002 Study. Med Clin (Barc), 122 (2004), pp. 165-171.

27. V. Barrios, M. Martínez, J.P. Tomás, I. Herranz, L. Prieto, J.L. Llisterri, et al. Perfil clínico de una población hipercolesterolémica española y diferencias entre sexos. Estudio LIPYCARE. Hipertensión, 21 (2004), pp. 395402.

28. F.J. García Ruiz, A. Marín Ibánez, F. Pérez-Jiménez, X. Pinto, G. Nocea, C. Ahumada, et al. Current lipid management and low cholesterol goal attainment in common daily practice in Spain. The REALITY Study Pharmacoeconomics, 22 (Suppl 3) (2004), pp. 1-12.

29. Grupo de Investigación del estudio ELIPSE (El Estudio de Lípidos en Prevención Secundaria de Cardiopatía Isquémica). Prevención secundaria de la cardiopatía isquémica en la provincia de Ciudad Real. Efectividad de la terapéutica hipolipemiante en atención primaria. Med Clin (Barc), 115 (2000), pp. 321-325.

30. Grupo Barbanza para el Estudio de las Enfermedades Cardiovasculares. Características de los pacientes con cardiopatías en un área sanitaria de la provincia de A Coruña. Estudio Barbanza 2000. Rev Clin Esp, 203 (2003), pp. $570-576$.

31. J.A. De Velasco, E. Llargues, R. Fito, J. Sala, A. del Rio, E. de Los Arcos, et al.. Prevalencia de los factores de riesgo y tratamiento farmacológico al alta hospitalaria en el paciente coronario. Resultados de un registro multicéntrico nacional (Programa 3C). Rev Esp Cardiol, 54 (2001), pp. 159-168.

32. J.A. De Velasco, J. Cosín, J.L. López-Sendón, E. De Teresa, M. De Oya, G. Sellers. Nuevos datos sobre la prevención secundaria del infarto de miocardio en España. Resultados del estudio PREVESE II. Rev Esp Cardiol, 55 (2002), pp. 801-809.

33. R.H. Chapman, A.A. Petrilla, S. Tang, L. Berman, J.S. Benner. Are high-risk hypertensive patients prescribed concomitant statin therapy? Prescription patterns in patients initiating antihypertensive therapy in the United States. J Am Coll Cardiol, 47 (Suppl A) (2006), p. 230A. 